\title{
Severe hypereosinophilic syndrome successfully treated with a monoclonal antibody against interleukin 5 receptor $\alpha$ - benralizumab
}

\author{
JOANNA KOSAEKA-WĘGIEL $L^{1,2}$, MAMERT MILEWSKI', ANDŻELIKA SIWIEC ${ }^{1,2}$, \\ MAGDALENA STRACH ${ }^{l}$, BOGDAN OCHREM ${ }^{3}$, MARIUSZ KORKOSZ ${ }^{l}$ \\ ${ }^{1}$ Department of Rheumatology and Immunology, Jagiellonian University Medical College, Kraków, Poland \\ ${ }^{2} 2^{\text {nd }}$ Department of Internal Medicine, University Hospital, Kraków, Poland \\ ${ }^{3}$ Department of Hematology, University Hospital, Kraków, Poland
}

\begin{abstract}
Hypereosinophilic syndrome (HES) is a group of a rare diseases characterized by marked eosinophilia in blood or tissue and eosinophil-related clinical manifestations. Benralizumab is a humanized, monoclonal antibody against interleukin $5(I L-5)$ receptor $\alpha$, which is expressed on human eosinophils.

Here, we present the case of a patient with severe HES in whom treatment with benralizumab, an anti-IL-5 receptor monoclonal antibody, was initiated 6 months ago. Prior to benralizumab administration, the patient was treated with glucocorticoids (GS) and mepolizumab. However, instead of the applied treatment and normal level of peripheral eosinophils the patient presented with fluctuating lower respiratory tract symptoms and recurrent exacerbations of HES.

Treatment with benralizumab (30 mg s.c. every 4-6 weeks) was started, resulting in significant improvement of respiratory signs and symptoms, normalization of eosinophil count and significant reduction of the methylprednisolone dose (after 5 doses of benralizumab administration). No substantial side effects have been noted during treatment and 6-month follow-up.

We argue that in the severe and relapsing course of HES, rescue treatment with benralizumab should be taken into account, particularly in cases of relative inefficacy of GS and mepolizumab.
\end{abstract}

Key words: hypereosinophilic syndrome, HES, benralizumab, IL-5, IL-5 receptor, monoclonal antibody.

(Cent Eur J Immunol 2021; 46 (3): 395-397)

\section{Introduction}

Hypereosinophilic syndrome (HES) is a group of rare chronic disorders characterized by an unexplained sustained elevated absolute eosinophil count (> 1500 cells/ $\mu \mathrm{l})$ and evidence of eosinophil-related clinical manifestations that can include intractable pruritus, pulmonary infiltrates, eosinophilic gastroenteritis, endomyocardial fibrosis, and thromboembolism [1-6]. The goal of treatment is a reduction in blood and tissue eosinophilia, thereby preventing organ damage and failure [1, 2]. Conventional therapies, including glucocorticoids (GS) and immunomodulatory and cytotoxic therapies, have variable efficacy and substantial side effects $[1,2]$. Interleukin 5 (IL-5) is the pivotal cytokine involved in the differentiation, maturation, migration, development, survival, trafficking and effector function of blood and local tissue eosinophils [7-9]. Therefore, therapies targeted on the IL-5 pathway seem to be an attractive extension in the treatment of patients with many hypereosinophilic conditions, including eosinophilic asthma, eosinophilic granulomato- sis with polyangiitis, eosinophilic nasal polyps or HES [7, 9, 10]. We present a case of idiopathic HES treated with the anti-IL-5 receptor monoclonal antibody benralizumab, resulting in significant clinical improvement of respiratory signs and symptoms, and normalization of eosinophil count.

\section{Case report}

In June 2017, a nonsmoker 48-year-old woman with a history of persistent bronchial asthma and chronic pansinusitis was diagnosed with respiratory failure in the course of hypereosinophilic syndrome. Computed tomography (CT) of the chest showed massive interstitial infiltrates and laboratory tests revealed eosinophilia $(8286$ cells/ $\mu$ l). Therapy with systemic and inhaled glucocorticoids and inhaled bronchodilators was started, with moderate improvement. Further workup displayed elevated level of eosinophils (8\%) in bronchoalveolar lavage (BAL) fluid and negative antinuclear and antineutrophil cytoplasmic antibodies. Previous

Correspondence: Joanna Kosałka-Węgiel, MD, PhD, Department of Rheumatology and Immunology, Jagiellonian University Medical College, 2 Jakubowskiego St., 30-688 Kraków, Poland, phone: +48 1240031 10, e-mail: joanna.kosalka@uj.edu.pl Submitted: 7.09.2020; Accepted: 3.01.2021 

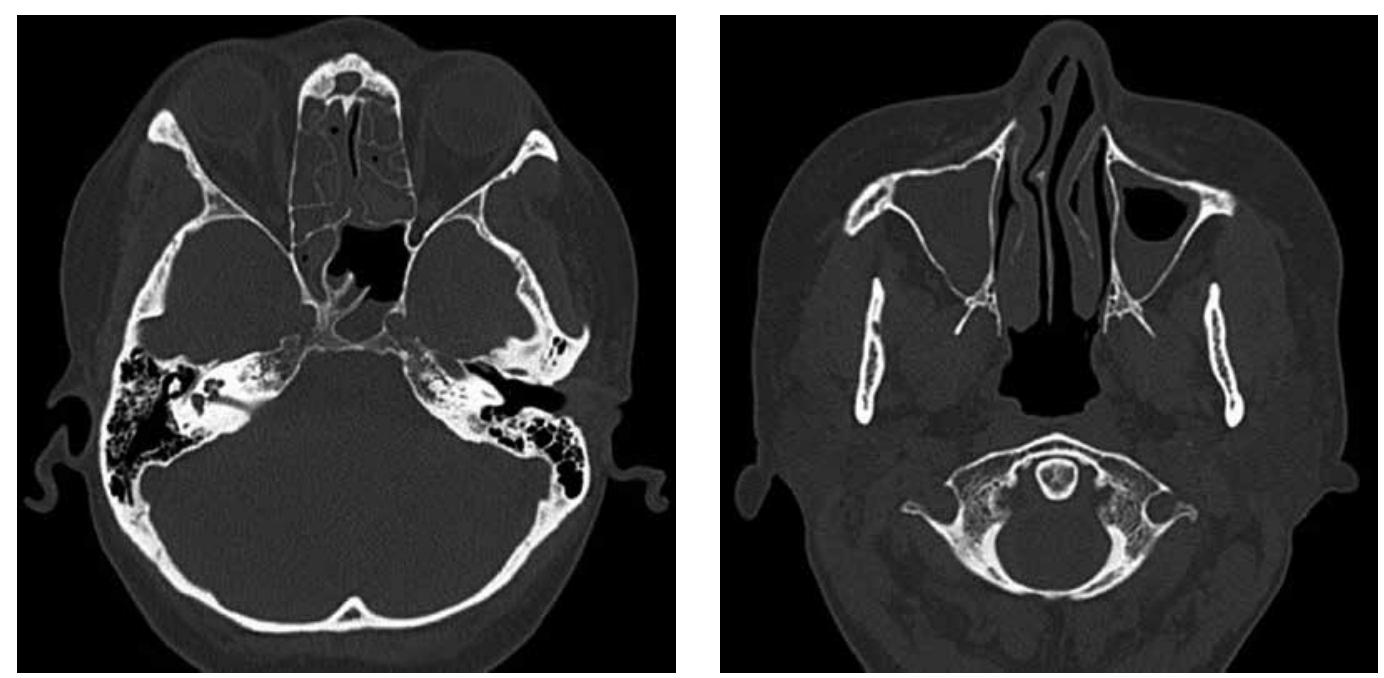

Fig. 1. CT scan: diffuse pansinusitis (12.06.2019)
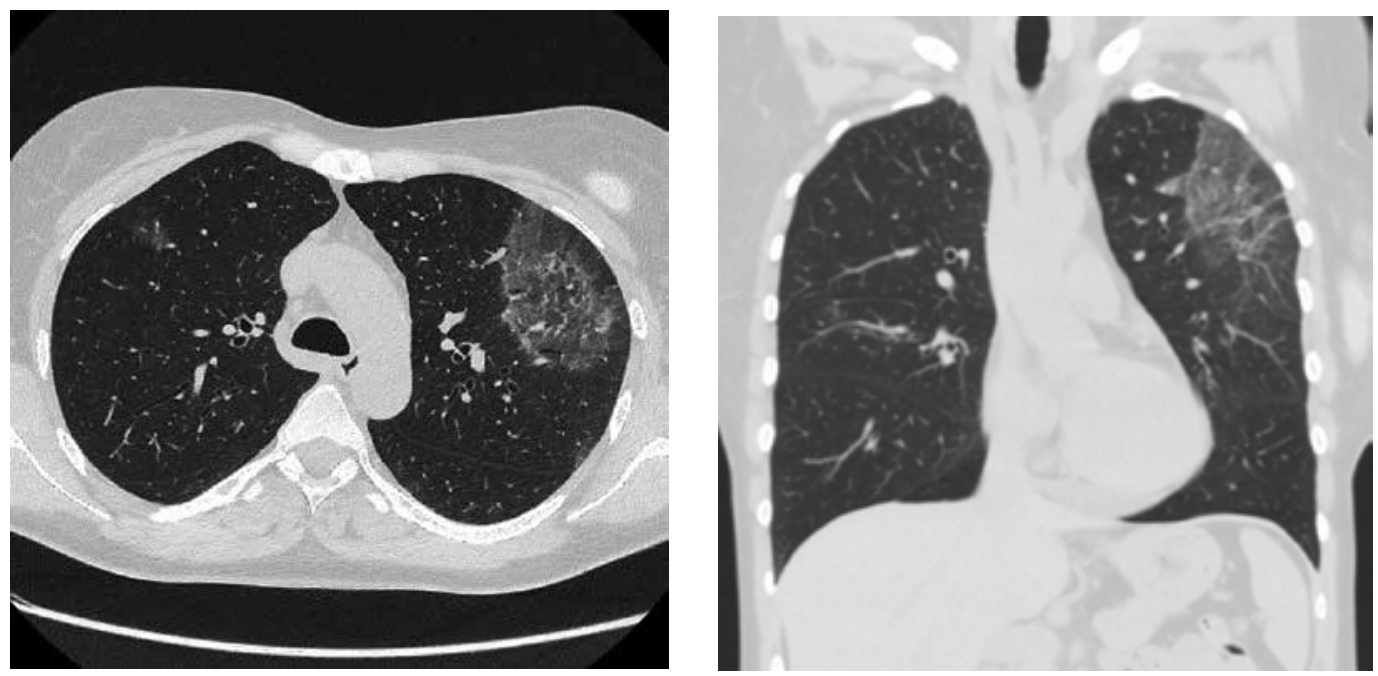

Fig. 2. Ground glass opacities on high-resolution CT (31.12.2019)

attempts to taper oral GS led to flare of the disease; therefore the patient had add-on therapy with the humanized anti-IL-5 monoclonal antibody mepolizumab for several months (last dose was given in May 2019). However, instead of the applied treatment and normal level of peripheral eosinophils $(<500$ cells/ $\mu \mathrm{l})$ she presented with fluctuating lower respiratory tract symptoms (wheezing, rhonchi, cough, dyspnea), recurrent exacerbations of pansinusitis with persisting hearing impairment (Fig. 1). In July 2019 functional endoscopic sinus surgery was performed, revealing eosinophilic (approx. $10 \%$ of the cells) chronic infiltrates of the mucosal stroma.

In December 2019 the patient was admitted to the Department of Rheumatology and Immunology due to exertional dyspnea and cough. Physical examination revealed diffuse expiratory wheezing and prolonged expiration. Initial workup revealed blood eosinophilia ( 2840 cells/ $\mu \mathrm{l})$ and an elevated level of immunoglobulin E (134 IU/ml, reference range $<100$ ); with normal levels of vitamin $B_{12}$, tryptase and immunoglobulin $\mathrm{G}$. There were no parasitic eggs or larvae in a stool sample. The patient was negative for FIP1L1-PDGFRA, JAK2 and ETV6-PDGFRB mutations. A bone marrow aspirate revealed an elevated level of eosinophils ( $<10 \%$ of the cells); no other pathologies were identified. Cytogenetic analysis showed a normal female karyotype. Ground glass opacities on high-resolution CT were described (Fig. 2). Bacterial and fungal cultures of BAL fluid did not yield any organisms. Increased eosinophils in BAL fluid (10\%) confirmed HES flare. Therapy with intravenous methylprednisolone (40 mg daily for 5 days, subsequently tapered), combined with inhaled GC and bronchodilators was started. Reduction of methylprednisolone dose less than $32 \mathrm{mg}$ daily led to HES flare. 


\section{Results}

Off-label treatment with benralizumab $(30 \mathrm{mg}$ s.c. every 4-6 weeks) 20.03.2020 was started, resulting in significant improvement of respiratory signs and symptoms, normalization of eosinophil count and reduction of methylprednisolone dose to $3 \mathrm{mg}$ daily (after 5 doses of benralizumab administration). No substantial side effects have been noted during treatment and 6-month follow-up.

\section{Discussion}

We believe that it is the first case report in Poland of successful treatment of HES with benralizumab. Benralizumab is a humanized, afucosylated, immunoglobulin (Ig) G1-type anti-IL-5 receptor monoclonal antibody which induces antibody-dependent cellular cytotoxicity (ADCC) against both eosinophils and basophils, executed by natural killers (NK) cells and/or macrophages [1, 7, 11-13]. Afucosylation of the antibody results in marked enhancement of its affinity for the FcyRIIIa receptor on NK cells, thereby competing with non-specific endogenous IgGs, and making benralizumab a highly efficient cytotoxic antibody [12]. Moreover, because of its mechanism of action, benralizumab can destroy IL- 5 receptor $\alpha$-expressing cells, regardless of their relative dependency on IL-5 or other mediators for their growth or survival [12]. Interestingly, ADCC is not significantly affected by the density of target antigen, so benralizumab is capable of destroying cells even if they display low-level expression of the IL-5 receptor $\alpha$ chain [12]. Importantly, preliminary data suggest that benralizumab depletes both mature eosinophils and their precursors in the bone marrow, and it may explain the prolonged eosinopenia observed after a single intravenous dose [13]. As a consequence, complete depletion of eosinophils in the blood and bone marrow within 24 hours after the first administration, and almost complete depletion in sputum and tissues $(90 \%$ and $96 \%$, respectively), have been reported [11]. So far, one double-blind trial with benralizumab enrolling 20 symptomatic patients with PDGFRA-negative HES has shown a significant clinical and laboratory improvement [1]. During the open-label phase, clinical and hematologic responses were observed in 17 of 19 patients (89\%) and were sustained for 48 weeks in 14 of 19 patients (74\%) [1]. Bone marrow and tissue eosinophilia were also suppressed with benralizumab therapy [1]. Benralizumab is effective in reducing blood and tissue eosinophilia with few or no adverse effects in patients with severe HES [1, 14].

\section{Conclusions}

In our case the efficacy and safety of the treatment with benralizumab have been proven. We argue that in the severe and relapsing course of HES, rescue treatment with benralizumab should be taken into account, particularly in cases of relative inefficacy of GS and mepolizumab.

The authors declare no conflict of interest.

\section{References}

1. Kuang FL, Legrand F, Makiya M, et al. (2019): Benralizumab for PDGFRA-negative hypereosinophilic syndrome. N Engl J Med 380: 1336-1346.

2. Klion AD (2015): How I treat hypereosinophilic syndromes. Blood 126: 1069-1077.

3. Gotlib J (2017): World Health Organization-defined eosinophilic disorders: 2017 update on diagnosis, risk stratification, and management. Am J Hematol 92: 1243-1259.

4. Kay D, Ataya A, Liu X, et al. (2018): Dysphonia and dyspnea in idiopathic hypereosinophilic syndrome treated with Mepolizumab. Respir Med Case Rep 25: 30-32.

5. Xie J, Zhang J, Zhang X, et al. (2020): Cough in hypereosinophilic syndrome: case report and literature review. BMC Pulm Med 20: 90.

6. Khalid F, Holguin F (2019): Hypereosinophilic syndrome in an elderly female: a case report. Am J Case Rep 20: 381-384.

7. FitzGerald JM, Bleecker ER, Nair P, et al. (2016): Benralizumab, an anti-interleukin-5 receptor $\alpha$ monoclonal antibody, as add-on treatment for patients with severe, uncontrolled, eosinophilic asthma (CALIMA): a randomised, double-blind, placebo-controlled phase 3 trial. Lancet 388: 2128-2141.

8. Liu W, Ma X, Zhou W (2019): Adverse events of benralizumab in moderate to severe eosinophilic asthma: A meta-analysis. Med 98: e15868.

9. Molfino NA, Gossage D, Kolbeck R, et al. (2012): Molecular and clinical rationale for therapeutic targeting of interleukin-5 and its receptor. Clin Exp Allergy 42: 712-737.

10. Hassani M, Koenderman L (2018): Immunological and hematological effects of IL-5(R $\alpha)$-targeted therapy: An overview. Allergy 73: 1979-1988.

11. Caminati M, Menzella F, Guidolin L, et al. (2019): Targeting eosinophils: severe asthma and beyond. Drugs Conte 8 : 212587.

12. Roufosse F (2018): Targeting the interleukin-5 pathway for treatment of eosinophilic conditions other than asthma. Front Med 5: 49.

13. Legrand F, Klion A (2015): Biologic therapies targeting eosinophils: current status and future prospects. J Allergy Clin Immunol Pr 3: 167-174.

14. Yamada H, Hida N, Kurashima Y, et al. (2019): A case of severe eosinophilic asthma and refractory rheumatoid arthritis well controlled by combination of IL-5R a antibody and TNF a inhibitor. Allergol Int 68: 536-538. 